\title{
Photon radiation in hot nuclear matter by means of chiral anomalies
}

\author{
Kirill Tuchin \\ Department of Physics and Astronomy, Iowa State University, Ames, Iowa 50011, USA
}

(Received 22 March 2019; published 24 June 2019)

\begin{abstract}
A new mechanism of photon emission in the quark-gluon plasma is proposed. Photon dispersion relation in the presence of the $C P$-odd topological regions generated by the chiral anomaly acquires an imaginary mass. It allows photon radiation through the decay $q \rightarrow q \gamma$ and annihilation $q \bar{q} \rightarrow \gamma$ processes closely related to the chiral Cherenkov radiation. Unlike previous proposals this mechanism does not require an external magnetic field. The differential photon emission rate per unit volume is computed and shown to be comparable to the rate of photon emission in conventional processes.
\end{abstract}

DOI: 10.1103/PhysRevC.99.064907

\section{INTRODUCTION}

Photon radiation by hot nuclear matter has been a focus of experimental and theoretical studies for many decades. However, in spite of considerable progress, there are still unresolved problems concerning the photon spectrum produced in relativistic heavy ion collisions, such as the puzzling enhancement of the direct photon production $[1,2]$. The major contributors to the photon spectrum are the quark-antiquark annihilation and the QCD Compton scattering processes in the quark-gluon plasma and the inelastic reactions in the hot hadronic gas [3-18]. In addition to these "conventional" processes, other contributors have been proposed, such as photon emission by the nuclear matter before the QGP formation $[19,20]$, the synchrotron radiation [21,22], radiation via the conformal anomaly [23] and through the chiral anomaly [24], as well as the modification of the conventional processes due to the axial charge fluctuations $[25,26]$. The mechanisms suggested in Refs. [21,23,24] rely on existence of intense magnetic field produced in heavy-ion collisions. In this article it is argued that there is a different unconventional mechanism of photon production via the chiral anomalies of QED and QCD which does not involve the external magnetic field.

The hot nuclear matter, or quark-gluon plasma (QGP), is believed to contain the topological $C P$-odd domains created by the random sphaleron-mediated transitions between different QCD vacua. Interaction of the electromagnetic field with these domains can be described by adding to the QED Lagrangian the axion-photon coupling term [27]

$$
\mathcal{L}_{A}=-\frac{c_{A}}{4} \theta F_{\mu \nu} \tilde{F}^{\mu \nu},
$$

Published by the American Physical Society under the terms of the Creative Commons Attribution 4.0 International license. Further distribution of this work must maintain attribution to the author(s) and the published article's title, journal citation, and DOI. Funded by $S C O A P^{3}$. where $c_{A}=N_{c} \sum_{f} q_{f}^{2} e^{2} / 2 \pi^{2}$ is the QED anomaly coefficient and the field $\theta$ is sourced by the topological charge density

$$
q(x)=\frac{g^{2}}{32 \pi^{2}} G_{\mu \nu}^{a} \tilde{G}^{a \mu \nu}(x),
$$

which varies in space and time across a $C P$-odd domain. As a result Eq. (1) cannot be rewritten as a total derivative and removed from the Lagrangian. Instead, it appears in the modified Maxwell equations as the spatial and the temporal derivatives of $\theta$.

It has been known since the pioneering article by Carroll, Feld, and Jackiw [28] that in QED coupled to the axion field, photons acquire an imaginary mass $m_{A}$ making possible their spontaneous emission by fermions. This phenomenon is referred to as the vacuum Cherenkov radiation [29,30]. Since the electromagnetic field in QGP is coupled to the axion-like field $\theta$, it is natural to expect that a similar mechanism of photon radiation exists in hot nuclear medium as well. This idea was developed in Refs. [31,32], where it was argued that ultrarelativistic fermions moving in a finite- $\theta$ domain radiate photons, which I dubbed the chiral Cherenkov radiation. Additionally, fermions in QGP radiate the chiral transition radiation as they cross the boundary between the plasma and vacuum due to the difference in the photon wave function inside and outside the plasma. The spectra of both processes are proportional to the average values of the spatial and the temporal $\theta$-derivatives. Since the chiral Cherenkov radiation scales with the system volume, whereas the chiral transition radiation scales with its area, the former is dominant when the contribution of the entire QGP (as opposed to a single fast quark) is considered. Thus, the present work focuses on the chiral Cherenkov radiation by QGP.

The analysis of Refs. [31,32] relied on two basic assumptions: (i) $\theta(x)$ is a slowly varying adiabatic function of its arguments and (ii) the absolute value of the photon mass generated by the anomaly $\left|m_{A}\right|$ is much larger than the plasma frequency $\omega_{\mathrm{pl}}$. The first assumption is the simplest model that captures the essential dynamics of the chiral magnetic effect [33-36]. It is supported by the results obtained by Zhitnitsky 
[37,38]. The second assumption is justified for large enough photon energy $\omega$ because $m_{A}^{2}$ is proportional to $\omega$, see Eq. (10), whereas the plasma frequency is $\omega$-independent. These are the assumptions that are carried over to the present study as well. However, unlike the radiation by a single quark discussed in Refs. [31,32] where one is free to choose the quark energy high enough so that most of the photon spectrum satisfy $\left|m_{A}\right| \gg \omega_{\mathrm{pl}}$, in the case of QGP the bulk of the photon radiation occurs at $\omega \lesssim T$, where $T$ is the QGP temperature. Still, it is argued in the next section that at high enough temperatures, the photon mass satisfies the second assumption since the plasma frequency is proportional to $T$, see Eq. (8), whereas the absolute value of $m_{A}^{2}$ is proportional to the sphaleron transition rate $\Gamma$ which rises at high temperatures as $T^{4}$.

The paper is organized as follows. Section II deals with the qualitative discussion of the electromagnetic fields in presence of the $C P$-odd domains. The mean value of the $\theta$-field in a domain is related to the sphaleron transition rate and hence scales as $T^{4}$ at high temperatures. This indicates that at high enough temperatures the chiral Cherenkov radiation becomes possible. In Sec. III, the photon dispersion relation at finite $\theta$ is discussed. The main section is Sec. IV, where the photon radiation rate is computed. To simplify the derivations and emphasize the main physics points, I am going to consider the relativistic limit $\omega \gg\left|m_{A}\right|$; generalization beyond this limit is straightforward. In fact, such a generalization for a single quark has been recently obtained in Ref. [39]. The discussion and summary is presented in Sec. V.

\section{ELECTRODYNAMICS IN QUARK-GLUON PLASMA WITH $C P$-ODD DOMAINS}

The $C P$-odd domains in the chiral matter can be described by a pseudoscalar field $\theta$ whose interactions with the electromagnetic $F_{\mu \nu}$ and color $G_{\mu \nu}^{a}$ fields are governed by the Lagrangian [28,40-42]

$$
\begin{aligned}
\mathcal{L}= & \mathcal{L}_{\mathrm{QED}}+\mathcal{L}_{\mathrm{QCD}}-\frac{c_{A}}{4} \theta F_{\mu \nu} \tilde{F}^{\mu \nu}-\frac{c_{A}^{\prime}}{4} \theta G_{\mu \nu}^{a} \tilde{G}^{a \mu \nu} \\
& +f^{2}\left[\frac{1}{2}\left(\partial_{\mu} \theta\right)^{2}-\frac{1}{2} m_{\mathrm{ax}}^{2} \theta^{2}\right],
\end{aligned}
$$

where $\tilde{F}_{\mu \nu}=\frac{1}{2} \epsilon_{\mu \nu \lambda \rho} F^{\lambda \rho}$ is the dual field tensor, $c_{A}, c_{A}^{\prime}$ are the QED and QCD anomaly coefficients, respectively, and $f, m_{\mathrm{ax}}$ are constants with mass dimension one. It follows that the equation of motion of the $\theta$-field is

$$
\left(\partial^{2}+m_{\mathrm{ax}}^{2}\right) \theta=-\frac{1}{4 f^{2}}\left(c_{A}^{\prime} G_{\mu \nu}^{a} \tilde{G}^{a \mu \nu}+c_{A} F_{\mu \nu} \tilde{F}^{\mu \nu}\right) .
$$

In the quark-gluon plasma the electromagnetic contribution to the topological charge density is presumed to be negligible so that the $\theta$-field dynamics is driven primarily by the topologically non-trivial gluon configurations. Assuming that $\theta$ is slowly varying inside a $C P$-odd domain one can express it in terms of the topological number density Eq. (2) as

$$
\theta(x)=-\frac{N_{f} q(x)}{f^{2} m_{\mathrm{ax}}^{2}} .
$$

The equations of motion of electromagnetic field read

$$
\begin{aligned}
& \partial_{\mu} F^{\mu \nu}=j^{v}-c_{A} \tilde{F}^{\mu \nu} \partial_{\mu} \theta, \\
& \partial_{\mu} \tilde{F}^{\mu \nu}=0 .
\end{aligned}
$$

In a slowly varying field $\theta$, its first derivatives $\partial^{\mu} \theta$ can be replaced by their constant domain-average values denoted by $\sigma_{\chi}=c_{A} \dot{\theta}[35,36,43]$, referred to as the chiral conductivity, and $\boldsymbol{b}=c_{A} \nabla \theta$. In this approximation the photon and the $\theta$ field dynamics decouple and one can consider electrodynamics in the topologically non-trivial background [44].

The average of the $\theta$-field over an ensemble of $C P$-odd domains vanishes. However, its value in a single domain can be finite due to the fluctuations of the topological number $N_{C S}$. In the context of this work one needs to know the temperature dependence of the $\theta$-field in a domain because it determines the temperature dependence of the effective photon mass $m_{A}$. In particular, if its $T$-dependence is steeper than linear, then one expects that there is a range of temperatures where the plasma becomes radioactive as explained at the end of Sec. I. The topological number density can be estimated as $q \sim$ $N_{C S} / V_{\text {dom }}$, where $V_{\text {dom }} \sim 1 / m_{\mathrm{ax}}^{4}$ is the domain 4-volume. Since the sphaleron size is inversely proportional to $T$, the domain volume decreases as $V_{\text {dom }} \sim 1 / T^{4}$. Fluctuations of $N_{C S}$ are related to the sphaleron transition rate $\Gamma$ as $\left\langle N_{C S}^{2}\right\rangle=2 \Gamma V_{\mathrm{pl}}$ [45] for large enough 4-volume $V_{\mathrm{pl}}$ of plasma. Therefore, the variance of the topological number density is $\left\langle q^{2}\right\rangle \sim m_{\mathrm{ax}}^{8} \Gamma V_{\mathrm{pl}}$. Employing Eq. (5) it is seen that the typical variance of the $\theta$-field strength is $\left\langle\theta^{2}\right\rangle \sim m_{\mathrm{ax}}^{4} \Gamma V_{\mathrm{pl}} / f^{4}$. $\Gamma$ is exponentially suppressed at low temperatures, but increases as $T^{4}$ at high temperatures [46-49]. It follows, using Eq. (10) of the next section, that $m_{A} \sim\langle\theta\rangle^{1 / 2} \sim T^{4}$. Thus, $\left|m_{A}\right|$ exceeds $\omega_{\mathrm{pl}}$ at high $T$ making the chiral Cherenkov radiation possible.

\section{PHOTON DISPERSION RELATION}

Now that the model parameters have been outlined, it is instructive to review the photon dispersion relation. In the case $\theta=0$ the photon dispersion relation at finite temperature $T$ and finite chemical potentials of the right and left-handed fermions $\mu_{R, L}$ was computed in Ref. [50]. In the high-energy limit, when the photon is near the mass-shell and transversely polarized, its dispersion relation is $\omega^{2}-k^{2}=\omega_{\mathrm{pl}}^{2}$, where

$$
\omega_{\mathrm{pl}}^{2}=\frac{m_{D}^{2}}{2}=\frac{e^{2}}{2}\left(\frac{T^{2}}{6}+\frac{\mu^{2}}{2 \pi^{2}}\right)
$$

and $\mu^{2}=\mu_{R}^{2}+\mu_{L}^{2}$

At finite $\theta$ the photon dispersion relation acquires an extra term due its interaction with the $C P$-odd domains

$$
\omega^{2}-k^{2}=\omega_{\mathrm{pl}}^{2}+m_{A}^{2}+\mathcal{O}(\omega-k),
$$

where $m_{A}^{2}$ is given by

$$
m_{A}^{2}=-\lambda \sigma_{\chi} \omega, \quad \text { or } \quad m_{A}^{2}=-\lambda \boldsymbol{k} \cdot \boldsymbol{b},
$$


depending on which of the parameters $\sigma_{\chi}$ or $b$ is largest [32] ${ }^{1}$ and $\lambda= \pm 1$ is the right- and left-handed photon polarization. Note that $m_{A}$ can be real or imaginary. As explained in the previous two sections, at high enough photon energies and plasma temperatures $\omega_{\mathrm{pl}}$ is but a small correction compared to $m_{A}$ and will be neglected in the following sections.

\section{PHOTON RADIATION RATE}

Photon emission by means of the chiral Cherenkov radiation mechanism can proceed via two channels: (i) the decay channel $q \rightarrow q \gamma$ and (ii) the annihilation channel $q \bar{q} \rightarrow \gamma \cdot{ }^{2}$ The total photon radiation rate is the sum of rates of these two processes.

\section{A. Decay channel}

The scattering matrix element for photon radiation in the decay channel $q(p) \rightarrow q\left(p^{\prime}\right)+\gamma(k)$ is given by $S_{D}=$ $(2 \pi)^{4} \delta^{(4)}\left(p^{\prime}+k-p\right) i \mathcal{M}_{D}$, where

$$
i \mathcal{M}_{D}=-i e Q \frac{\bar{u}_{\boldsymbol{p}^{\prime} s^{\prime}} \notin_{\boldsymbol{k} \lambda}^{*} u_{\boldsymbol{p s}}}{\sqrt{8 \varepsilon \varepsilon^{\prime} \omega V^{3}}} .
$$

The components of the 4-vectors are $p=(\varepsilon, \boldsymbol{p}), p^{\prime}=\left(\varepsilon^{\prime}, \boldsymbol{p}^{\prime}\right)$, and $k=(\omega, \boldsymbol{k}), Q$ is quark charge, and $m=g T / \sqrt{3}$ its thermal mass [11]. I retained the relativistic normalization factors $\left(2 p^{0} V\right)^{-1 / 2}$ for each of the three fields, where $V$ is the normalization volume. The radiation probability can be computed as

$$
d w_{D}=2 N_{c} \frac{1}{2} \sum_{\lambda s s^{\prime}}\left|S_{D}\right|^{2} f(\varepsilon)\left[1-f\left(\varepsilon^{\prime}\right)\right] \frac{V d^{3} p^{\prime}}{(2 \pi)^{3}} \frac{V d^{3} k}{(2 \pi)^{3}} \frac{V d^{3} p}{(2 \pi)^{3}},
$$

where $2 N_{c}$ accounts for the number quarks and antiquarks of different color, $1 / 2$ comes from the incident quark spin average and $f(\varepsilon)$ is the quark equilibrium distribution function, which reads

$$
f(\varepsilon)=\frac{1}{e^{\varepsilon / T}+1} .
$$

The small chemical potentials of quarks is neglected. The rate of photon production per unit volume can be computed as

$$
\begin{aligned}
d \Gamma_{D}= & \frac{d w_{D}}{V T}=2 N_{c} \frac{\delta\left(\omega+\varepsilon^{\prime}-\varepsilon\right)}{16(2 \pi)^{5} \varepsilon \varepsilon^{\prime} \omega} \\
& \times \sum_{\lambda s s^{\prime}}\left|i \mathcal{M}_{D}\right|^{2} f(\varepsilon)\left[1-f\left(\varepsilon^{\prime}\right)\right] d^{3} k d^{3} p .
\end{aligned}
$$

Performing the summation over the transverse photon polarizations using

$$
\sum_{\lambda} \epsilon_{k \lambda}^{\mu} \epsilon_{k \lambda}^{\nu *}= \begin{cases}0, & v \mu=0, \\ \delta^{i j}-\frac{k^{i} k^{j}}{k^{2}}, & v=i, \mu=j\end{cases}
$$

\footnotetext{
${ }^{1}$ In Ref. [32] $m_{A}$ was denoted as $\mu$. The dispersion relations for arbitrary $\sigma_{\chi}$ and $b$ can be found in Ref. [28].

${ }^{2}$ I am using the term "the chiral Cherenkov radiation" with respect to both channels.
}

yields the result

$$
\sum_{s s^{\prime}}\left|\mathcal{M}_{D}\right|^{2}=4\left[\varepsilon \varepsilon^{\prime}-m^{2}-\frac{(\boldsymbol{k} \cdot \boldsymbol{p})\left(\boldsymbol{k} \cdot \boldsymbol{p}^{\prime}\right)}{\boldsymbol{k}^{2}}\right] .
$$

In the high-energy limit the momenta of the initial and final quarks and the photon have a large component, say along the $z$ direction, that allows one to approximate

$$
\begin{aligned}
& p_{z} \approx \varepsilon\left(1-\frac{p_{\perp}^{2}+m^{2}}{2 \varepsilon^{2}}\right), \quad k_{z} \approx \omega\left(1-\frac{k_{\perp}^{2}+m_{A}^{2}}{2 \omega^{2}}\right), \\
& p_{z}^{\prime} \approx \varepsilon^{\prime}\left(1-\frac{p_{\perp}^{\prime 2}+m^{2}}{2 \varepsilon^{\prime 2}}\right) .
\end{aligned}
$$

Denoting by $x=\omega / \varepsilon$ the fraction of the incident quark energy carried away by the photon and substituting Eq. (17) into Eq. (15) one derives

$$
\sum_{s s^{\prime}}\left|\mathcal{M}_{D}\right|^{2}=\frac{2}{x^{2}(1-x)}\left[q_{\perp}^{2}\left(2-2 x+x^{2}\right)+m^{2} x^{4}\right],
$$

where $\boldsymbol{q}_{\perp}=x \boldsymbol{p}_{\perp}-\boldsymbol{k}_{\perp}$. In the same approximation the energy $\delta$ function can be written as

$$
\delta\left(\omega+\varepsilon^{\prime}-\varepsilon\right) \approx 2 x(1-x) \varepsilon \delta\left(q_{\perp}^{2}+m_{A}^{2}(1-x)+m^{2} x^{2}\right) .
$$

Substituting Eqs. (18) and (19) into Eq. (14) and integrating over $q_{\perp}$ instead of $p_{\perp}$ one finds

$$
\begin{aligned}
\omega \frac{d \Gamma_{D}}{d^{3} k}= & 2 N_{c} \frac{e^{2} Q^{2} \pi}{4(2 \pi)^{5}} \int_{0}^{1} \frac{d x}{x^{4}} f\left(\frac{\omega}{x}\right)\left[1-f\left(\frac{\omega(1-x)}{x}\right)\right] \\
& \times \sum_{\lambda}\left\{-m_{A}^{2}\left[(1-x)^{2}+1\right]-2 m^{2} x^{2}\right\} \theta\left(-\kappa_{\lambda}\right),
\end{aligned}
$$

where it is denoted

$$
\kappa_{\lambda}=m_{A}^{2}(1-x)+m^{2} x^{2} .
$$

Evidently, since $m^{2}>0$ the nonvanishing contribution to the photon production rate in this channel exists only if $m_{A}^{2}<0$. Moreover, $\kappa_{\lambda}$ is negative only if $\left|m_{A}^{2}\right|(1-x)>x^{2} m^{2}$, which occurs when

$$
0 \leqslant x<\frac{\left|m_{A}^{2}\right|}{2 m^{2}}\left(\sqrt{1+\frac{4 m^{2}}{\left|m_{A}^{2}\right|}}-1\right) .
$$

One can perform the integration of $x$ explicitly in the limit $m \ll\left|m_{A}\right|$. It is convenient to introduce a new variable $\xi=$ $1 / x-1$ in place of $x$ and rewrite Eq. (20) as

$$
\begin{aligned}
\omega \frac{d \Gamma_{D}}{d^{3} k}= & 2 N_{c} \frac{e^{2} Q^{2}}{8(2 \pi)^{4}} \int_{0}^{\infty} d \xi\left\{-m_{A}^{2}\left[\xi^{2}+(1-\xi)^{2}\right]\right. \\
& \left.-2 m^{2}\right\} f(\omega(1+\xi))[1-f(\omega \xi)] \\
& \times \theta\left(-m_{A}^{2} \xi(1+\xi)-m^{2}\right)
\end{aligned}
$$

where only the photon polarization that gives $m_{A}^{2}<0$ contributes. Neglecting $m$ one obtains

$$
\begin{aligned}
\omega \frac{d \Gamma_{D}}{d^{3} k}= & 2 N_{c} \frac{e^{2} Q^{2}}{8(2 \pi)^{4}}\left|m_{A}^{2}\right| \int_{0}^{\infty} d \xi\left[\xi^{2}+(1-\xi)^{2}\right] \\
& \times f(\omega(1+\xi))[1-f(\omega \xi)] .
\end{aligned}
$$


Note that the condition Eq. (22) is now trivial $0<x<1$. Also, since $e \ll g, \omega_{\mathrm{pl}} \ll m$ implying that $m_{A} \approx m_{A}$ in this approximation. One can also approximate $1-f(\omega \xi) \approx 1-$ $(e+1)^{-1}=0.73$ since the argument of $f$ is typically on the order of unity, for otherwise the distribution $f[\omega(1+\xi)]$ of the incident quark is exponentially suppressed. Thus, one derives

$$
\begin{aligned}
\omega \frac{d \Gamma_{D}}{d^{3} k}= & 0.73 \times 2 N_{c} \frac{e^{2} Q^{2}}{8(2 \pi)^{4}}\left|m_{A}^{2}\right|\left[\frac{\ln \left(1+e^{-\beta \omega}\right)}{\beta \omega}\right. \\
& \left.+\frac{2 \operatorname{Li}_{2}\left(-e^{-\beta \omega}\right)}{(\beta \omega)^{2}}-\frac{4 \operatorname{Li}_{3}\left(-e^{-\beta \omega}\right)}{(\beta \omega)^{3}}\right] .
\end{aligned}
$$

The low- and high-energy regions of the spectrum read

$$
\omega \frac{d \Gamma_{D}}{d^{3} k}=0.73 \times 2 N_{c} \frac{e^{2} Q^{2}}{8(2 \pi)^{4}}\left|m_{A}^{2}\right| \begin{cases}\frac{3 \zeta(3)}{(\beta \omega)^{3}}, & \omega \ll T, \\ \frac{1}{\beta \omega} e^{-\beta \omega}, & \omega \gg T .\end{cases}
$$

Taking into account that $m_{A}^{2}$ is proportional to $\omega$, one finds that at $\omega \ll T$, the photon of spectrum scales as $1 / \omega^{2}$. Thus, the total photon rate $\Gamma_{D}$ is dominated by soft photons $\omega \ll T$ that produce the large logarithm $\ln (T / m)$.

\section{B. Annihilation channel}

The scattering matrix element for photon radiation in the annihilation channel $q(p)+\bar{q}\left(p_{1}\right) \rightarrow \gamma(k)$ is given by $S_{A}=$ $(2 \pi)^{4} \delta^{(4)}\left(p+p_{1}-k\right) i \mathcal{M}_{A}$, where

$$
i \mathcal{M}_{A}=-i e Q \frac{\bar{v}_{p_{p_{1} s_{1}} \ell_{k \lambda}^{*} u_{p s}}}{\sqrt{8 \varepsilon \varepsilon_{1} \omega V^{3}}} .
$$

The corresponding radiation probability can be computed as

$$
d w_{A}=N_{c} \frac{1}{4} \sum_{\lambda s s^{\prime}}\left|S_{A}\right|^{2} f(\varepsilon) f\left(\varepsilon_{1}\right) \frac{V d^{3} p_{1}}{(2 \pi)^{3}} \frac{V d^{3} k}{(2 \pi)^{3}} \frac{V d^{3} p}{(2 \pi)^{3}},
$$

where $N_{c}$ accounts for different colors and 1/4 stems from the incident quark and antiquark spin average. The rate of photon production per unit volume reads

$$
\begin{aligned}
d \Gamma_{A}= & \frac{d w_{A}}{V T}=N_{c} \frac{\delta\left(\omega-\varepsilon_{1}-\varepsilon\right)}{32(2 \pi)^{5} \varepsilon \varepsilon_{1} \omega} \\
& \times \sum_{\lambda s s_{1}}\left|i \mathcal{M}_{A}\right|^{2} f(\varepsilon) f\left(\varepsilon_{1}\right) d^{3} k d^{3} p .
\end{aligned}
$$

Summation over the transverse photon polarizations using Eq. (15) yields

$$
\sum_{s s_{1}}\left|\mathcal{M}_{A}\right|^{2}=4\left[\varepsilon \varepsilon_{1}+m^{2}-\frac{(\boldsymbol{k} \cdot \boldsymbol{p})\left(\boldsymbol{k} \cdot \boldsymbol{p}_{1}\right)}{\boldsymbol{k}^{2}}\right] .
$$

Employing the high-energy limit Eq. (17) and denoting by $y=$ $\varepsilon / \omega$ the energy fraction that the incident quark contributed to the photon energy and $\boldsymbol{\ell}_{\perp}=y \boldsymbol{k}_{\perp}-\boldsymbol{p}_{\perp}$ one derives

$$
\sum_{s s_{1}}\left|\mathcal{M}_{A}\right|^{2}=\frac{2}{y(1-y)}\left\{\ell_{\perp}^{2}\left[y^{2}+(1-y)^{2}\right]+m^{2}\right\},
$$

and

$$
\delta\left(\omega-\varepsilon_{1}-\varepsilon\right) \approx 2 y(1-y) \omega \delta\left[\ell_{\perp}^{2}-m_{A}^{2} y(1-y)+m^{2}\right] .
$$

These formulas can also be obtained from the results of the previous subsection using the crossing-symmetry. Substituting Eqs. (31) and (32) into Eq. (29) and integrating over $\ell_{\perp}$ instead of $p_{\perp}$ one finds

$$
\begin{aligned}
\omega \frac{d \Gamma_{A}}{d^{3} k}= & N_{c} \frac{e^{2} Q^{2} \pi}{8(2 \pi)^{5}} \int_{0}^{1} d y f(y \omega) f[(1-y) \omega] \\
& \times \sum_{\lambda}\left[m_{A}^{2}\left(2 y^{2}-2 y+1\right)+2 m^{2}\right] \theta\left(-\varkappa_{\lambda}\right),
\end{aligned}
$$

where it is denoted

$$
\varkappa_{\lambda}=-m_{A}^{2} y(1-y)+m^{2} .
$$

In the annihilation channel $m_{A}^{2}$ must be positive in order that $\varkappa_{\lambda}$ be negative. Additionally, the energy fraction $y$ is restricted to the interval

$$
\frac{1}{2}\left(1-\sqrt{1-\frac{4 m^{2}}{\left|m_{A}^{2}\right|}}\right)<y<\frac{1}{2}\left(1+\sqrt{1-\frac{4 m^{2}}{\left|m_{A}^{2}\right|}}\right) .
$$

Clearly, the radiation is possible only if $\left|m_{A}\right|>2 m$.

In the limit $\left|m_{A}\right| \gg m$, Eq. (33) simplifies

$$
\begin{aligned}
\omega \frac{d \Gamma_{A}}{d^{3} k}= & N_{c} \frac{e^{2} Q^{2}}{16(2 \pi)^{4}}\left|m_{A}^{2}\right| \int_{0}^{1} d y f(y \omega) \\
& \times f[(1-y) \omega]\left(2 y^{2}-2 y+1\right),
\end{aligned}
$$

where only the photon polarization that gives $m_{A}^{2}>0$ contributes. The integral can be taken exactly:

$$
\begin{aligned}
& \int_{0}^{1} d y f(y \omega) f[(1-y) \omega]\left(2 y^{2}-2 y+1\right) \\
= & \frac{1}{e^{\beta \omega}-1}\left[-\frac{8 \operatorname{Li}_{3}\left(-e^{\beta \omega}\right)+6 \zeta(3)}{(\beta \omega)^{3}}\right. \\
& \left.+\frac{4 \operatorname{Li}_{2}\left(-e^{\beta \omega}\right)-\pi^{2} / 3}{(\beta \omega)^{2}}+\frac{2 \ln \left(1+e^{\beta \omega}\right)-\ln 4}{\beta \omega}-\frac{2}{3}\right] .
\end{aligned}
$$

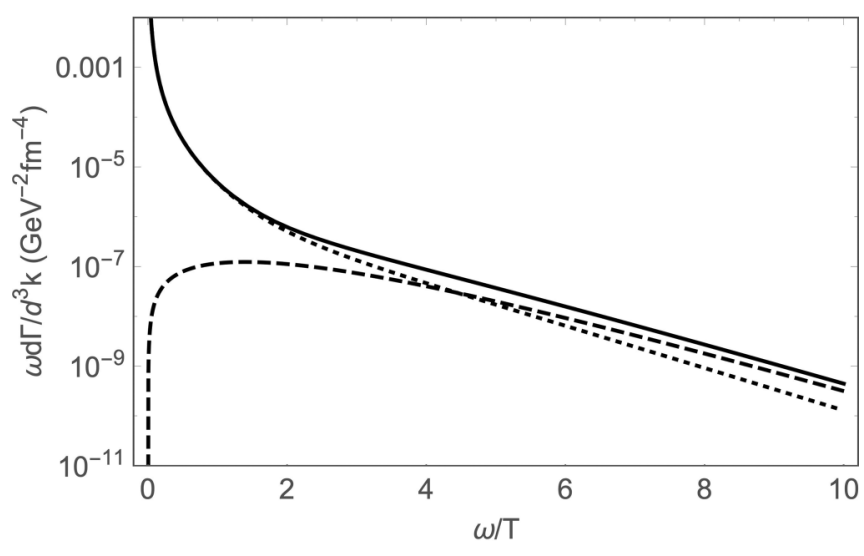

FIG. 1. Differential photon emission rate (solid line) and its two contributions from the decay (dashed line) and annihilation (dotted line) processes. Plasma temperature $T=400 \mathrm{MeV}$, chiral conductivity $\sigma_{\chi}=1 \mathrm{MeV}$ and $\sum_{f} Q_{f}^{2}=5 / 9$ (for the two lightest flavors). 
At low and high photon energy the spectrum reads

$$
\omega \frac{d \Gamma_{A}}{d^{3} k}=N_{c} \frac{e^{2} Q^{2}}{16(2 \pi)^{4}}\left|m_{A}^{2}\right| \begin{cases}\frac{1}{6}, & \omega \ll T, \\ \frac{2}{3} e^{-\beta \omega}, & \omega \gg T .\end{cases}
$$

Comparing with Eq. (26) one can see that the decay channel dominates the low-energy part of the spectrum, whereas the annihilation channel dominates the high-energy tail; see Fig. 1. It is remarkable that since the photon polarization in the two channels is opposite, the total photon spectrum has different polarization direction at low and high energies with respect to $T$.

\section{DISCUSSION AND SUMMARY}

The main result of this paper are Eqs. (20) and (33) that represent the differential rates of photon emission rate by means of the chiral Cherenkov radiation in the decay and annihilation channels. Their sum gives the total photon emission rate (per unit volume). The magnitude of this contribution to the total photon yield by QGP shown in Fig. 1 is comparable with the conventional contributions as one can glean from Fig. 3 of Ref. [17].

An important phenomenological question is the value of the photon emission threshold at a given QGP temperature. Electromagnetic radiation by means of the mechanism described in this paper is possible if $\omega_{\mathrm{pl}}<\left|m_{A}\right|$. The plasma frequency Eq. (8) of QGP at temperature $T=400 \mathrm{MeV}$ is $\omega_{\mathrm{pl}} \approx 35 \mathrm{MeV}$. The chiral conductivity is unknown, but is often estimated to be of the order of a fraction of $\mathrm{MeV}$. Importantly, it rapidly increases as $T^{4}$. Thus, for example, if $\sigma_{\chi}=1-10 \mathrm{MeV}$ then using the first of the Eqs. (10), the infrared photon emission threshold is $\omega_{0} \sim 0.1-1 \mathrm{GeV}$. This is certainly within the range of phenomenologically interesting photon energies. A more precise knowledge of $\sigma_{\chi}$ may be extracted from the measurements of the charge separation effect in relativistic heavy-ion collisions because this effect is generated by the anomalous electric current proportional to $\sigma_{\chi}[34]$.

The calculations performed in this paper relied on the high-energy approximation in which $\omega \gg m$. Since the quark thermal mass $m$ is of the order of a hundred $\mathrm{MeV}$, this approximation is not sufficiently reliable for the phenomenological applications to the QGP at realistic temperatures. Still, considering this limit has a great advantage of emphasizing the physics mechanism of photon radiation with the least mathematical and numerical complications possible. A comprehensive phenomenological approach would of course require going beyond the high-energy approximation.

\section{ACKNOWLEDGMENT}

This work was supported in part by the U.S. Department of Energy under Grant No. DE-FG02-87ER40371.
[1] A. Adare et al. (PHENIX Collaboration), Enhanced Production of Direct Photons in Au+Au Collisions at $\sqrt{s_{N N}}=200 \mathrm{GeV}$ and Implications for the Initial Temperature, Phys. Rev. Lett. 104, 132301 (2010).

[2] J. Adam et al. (ALICE Collaboration), Direct photon production in $\mathrm{Pb}-\mathrm{Pb}$ collisions at $\sqrt{s_{\mathrm{NN}}}=2.76 \mathrm{TeV}$, Phys. Lett. B 754, 235 (2016).

[3] R. Baier, H. Nakkagawa, A. Niegawa and K. Redlich, Production rate of hard thermal photons and screening of quark mass singularity, Z. Phys. C 53, 433 (1992).

[4] J. I. Kapusta, P. Lichard and D. Seibert, High-energy photons from quark-gluon plasma versus hot hadronic gas, Phys. Rev. D 44, 2774 (1991); 47, 4171(E) (1993).

[5] C. M. Hung and E. V. Shuryak, Dilepton/photon production in heavy ion collisions, and the QCD phase transition, Phys. Rev. C 56, 453 (1997).

[6] J. V. Steele, H. Yamagishi and I. Zahed, Dilepton and photon emission rates from a hadronic gas, Phys. Lett. B 384, 255 (1996).

[7] J. V. Steele, H. Yamagishi and I. Zahed, Dilepton and photon emission rates from a hadronic gas. II, Phys. Rev. D 56, 5605 (1997).

[8] K. Dusling and I. Zahed, Thermal photons from heavy ion collisions: A spectral function approach, Phys. Rev. C 82, 054909 (2010).

[9] C. H. Lee, J. Wirstam, I. Zahed and T. H. Hansson, Thermal dileptons from a nonperturbative quark - gluon phase, Phys. Lett. B 448, 168 (1999).
[10] P. Aurenche, F. Gelis and H. Zaraket, Landau-PomeranchukMigdal effect in thermal field theory, Phys. Rev. D 62, 096012 (2000).

[11] P. B. Arnold, G. D. Moore and L. G. Yaffe, Photon emission from quark gluon plasma: Complete leading order results, J. High Energy Phys. 12 (2001) 009.

[12] T. Peitzmann and M. H. Thoma, Direct photons from relativistic heavy ion collisions, Phys. Rep. 364, 175 (2002).

[13] S. Turbide, R. Rapp and C. Gale, Hadronic production of thermal photons, Phys. Rev. C 69, 014903 (2004).

[14] E. L. Bratkovskaya, S. M. Kiselev and G. B. Sharkov, Direct photon production from hadronic sources in high-energy heavyion collisions, Phys. Rev. C 78, 034905 (2008).

[15] I. Vitev and B. W. Zhang, A Systematic study of direct photon production in heavy ion collisions, Phys. Lett. B 669, 337 (2008).

[16] H. van Hees, C. Gale and R. Rapp, Thermal photons and collective flow at the relativistic heavy-ion collider, Phys. Rev. C 84, 054906 (2011).

[17] J. F. Paquet, C. Shen, G. S. Denicol, M. Luzum, B. Schenke, S. Jeon and C. Gale, Production of photons in relativistic heavyion collisions, Phys. Rev. C 93, 044906 (2016).

[18] O. Linnyk, V. Konchakovski, T. Steinert, W. Cassing and E. L. Bratkovskaya, Hadronic and partonic sources of direct photons in relativistic heavy-ion collisions, Phys. Rev. C 92, 054914 (2015).

[19] M. Chiu, T. K. Hemmick, V. Khachatryan, A. Leonidov, J. Liao and L. McLerran, Production of photons and dileptons in the glasma, Nucl. Phys. A 900, 16 (2013). 
[20] L. McLerran and B. Schenke, The Glasma, Photons and the implications of anisotropy, Nucl. Phys. A 929, 71 (2014).

[21] K. Tuchin, Electromagnetic radiation by quark-gluon plasma in a magnetic field, Phys. Rev. C 87, 024912 (2013).

[22] H. U. Yee, Flows and polarization of early photons with magnetic field at strong coupling, Phys. Rev. D 88, 026001 (2013).

[23] G. Basar, D. E. Kharzeev, and V. Skokov, Conformal Anomaly as a Source of Soft Photons in Heavy Ion Collisions, Phys. Rev. Lett. 109, 202303 (2012).

[24] K. Fukushima and K. Mameda, Wess-Zumino-Witten action and photons from the chiral magnetic effect, Phys. Rev. D 86, 071501(R) (2012).

[25] K. A. Mamo and H. U. Yee, Spin polarized photons and dileptons from axially charged plasma, Phys. Rev. D 88, 114029 (2013).

[26] K. A. Mamo and H. U. Yee, Spin polarized photons from an axially charged plasma at weak coupling: Complete leading order, Phys. Rev. D 93, 065053 (2016).

[27] K. Fujikawa and H. Suzuki, Path Integrals and Quantum Anomalies (Clarendon, Oxford, UK, 2004), p. 284.

[28] S. M. Carroll, G. B. Field and R. Jackiw, Limits on a Lorentz and parity violating modification of electrodynamics, Phys. Rev. D 41, 1231 (1990).

[29] R. Lehnert and R. Potting, Vacuum Cherenkov Radiation, Phys. Rev. Lett. 93, 110402 (2004).

[30] R. Lehnert and R. Potting, Čerenkov effect in Lorentz-violating vacua, Phys. Rev. D 70, 125010 (2004); 70, 129906(E) (2004).

[31] K. Tuchin, Radiative instability of quantum electrodynamics in chiral matter, Phys. Lett. B 786, 249 (2018).

[32] X. G. Huang and K. Tuchin, Transition Radiation as a Probe of the Chiral Anomaly, Phys. Rev. Lett. 121, 182301 (2018).

[33] D. Kharzeev, Parity violation in hot QCD: Why it can happen, and how to look for it, Phys. Lett. B 633, 260 (2006).

[34] D. Kharzeev and A. Zhitnitsky, Charge separation induced by P-odd bubbles in QCD matter, Nucl. Phys. A 797, 67 (2007).

[35] K. Fukushima, D. E. Kharzeev and H. J. Warringa, The chiral magnetic effect, Phys. Rev. D 78, 074033 (2008).
[36] D. E. Kharzeev, Topologically induced local $\mathrm{P}$ and CP violation in QCD × QED, Annals Phys. 325, 205 (2010).

[37] A. R. Zhitnitsky, The topological long range order in QCD. Applications to heavy ion collisions and cosmology, EPJ Web Conf. 95, 03041 (2015).

[38] A. R. Zhitnitsky, Topological order and Berry connection for the Maxwell Vacuum on a four-torus, Phys. Rev. D 90, 105007 (2014).

[39] K. Tuchin, Chiral Cherenkov and chiral transition radiation in anisotropic matter, Phys. Rev. D 98, 114026 (2018).

[40] F. Wilczek, Two Applications of Axion Electrodynamics, Phys. Rev. Lett. 58, 1799 (1987).

[41] P. Sikivie, On the interaction of magnetic monopoles with axionic domain walls, Phys. Lett. B 137, 353 (1984).

[42] T. Kalaydzhyan, Chiral superfluidity of the quark-gluon plasma, Nucl. Phys. A 913, 243 (2013).

[43] D. E. Kharzeev and H. J. Warringa, Chiral magnetic conductivity, Phys. Rev. D 80, 034028 (2009).

[44] D. E. Kharzeev, The chiral magnetic effect and anomalyinduced transport, Prog. Part. Nucl. Phys. 75, 133 (2014).

[45] V. A. Rubakov and M. E. Shaposhnikov, Electroweak baryon number nonconservation in the early universe and in highenergy collisions, Usp. Fiz. Nauk 166, 493 (1996); Phys. Usp. 39, 461 (1996).

[46] G. D. Moore, C. Hu, and B. Muller, Chern-Simons number diffusion with hard thermal loops, Phys. Rev. D 58, 045001 (1998).

[47] D. Bodeker, G. D. Moore and K. Rummukainen, Chern-Simons number diffusion and hard thermal loops on the lattice, Phys. Rev. D 61, 056003 (2000).

[48] G. D. Moore and M. Tassler, The Sphaleron rate in SU(N) gauge theory, J. High Energy Phys. 02 (2011) 105.

[49] D. T. Son and A. O. Starinets, Minkowski space correlators in AdS/CFT correspondence: Recipe and applications, J. High Energy Phys. 09 (2002) 042.

[50] Y. Akamatsu and N. Yamamoto, Chiral Plasma Instabilities, Phys. Rev. Lett. 111, 052002 (2013). 